\title{
Aufbereitung von Instrumenten der Minimal Invasiven Chirurgie - Stand und Perspektiven
}

\author{
Ch. Lösche, U. Boenick \\ Institut für Mikro- und Medizintechnik, Technische Universität Berlin \\ E-mail: loesche@kf.tu-berlin.de
}

\section{EINLEITUNG}

Die Miniaturisierung der Instrumente der Minimal Invasiven Chirurgie hat Auswirkungen hinsichtlich Funktion, Verschleiß und Reinigungsfähigkeit. Speziell die Aufbereitung der Instrumente wird dadurch problematischer. Trotz des verstärkten Einsatzes von immer besseren Reinigungsautomaten ist bei einer zunehmenden Zahl von Instrumenten zu befürchten, dass der sich aus Reinigung, Desinfektion und wo vorgeschrieben Sterilisation zusammensetzende Prozeß nicht mit völliger Sicherheit durchgeführt werden kann.

Sowohl die Entfernung der Anschmutzung als auch die Prüfung des Reinigungserfolges ist bedingt durch kleine Lumen, filigrane Gelenke und kompliziert aufgebaute mechanische Bauteile zunehmend erschwert. Die bisher angewendeten visuell-taktilen Methoden zur Prüfung der Reinigungserfolges genügen - obwohl Stand der Technik - infolge einer subjektiven Urteilsfindung nicht den Anforderungen an eine Reproduzierbarkeit der Ergebnisse. Allein durch die fehlende Objektivität des Prüfers sind sie auch vor dem Hintergrund einer Validierung der Reinigungsverfahren nicht akzeptabel.

Problematisch ist, dass es noch kein allgemein akzeptiertes und praktikables Verfahren zur Prüfung der Reinigungsleistung bei Instrumenten der Minimal Invasiven Chirurgie gibt. Speziell fehlen Empfehlungen zur Routinekontrolle. So lässt sich die oben genannte Vermutung der möglicherweise unzureichenden Reinigung weder bestätigen noch widerlegen.

Zudem existiert kein Konsens darüber, was ,sauber" und was ,rein” bedeutet. Welcher Grad der Restkontamination ist tolerierbar? Daraus ergibt sich dann die Frage nach der Nachweisgrenze und der Genauigkeit der Prüfverfahren.

\section{MATERIAL UND METHODEN}

Unter dem Begriff der Aufbereitung von Instrumenten ist die vollständige Wiederherstellung der Einsatzfähigkeit im Sinne der ursprünglichen $Z_{\text {weckbestimmung zu }}$ verstehen. Im einzelnen kann dieser Vorgang insbesondere die Prozesse der Reinigung, Desinfektion und der 1
Sterilisation umfassen.

Dabei führt allein die Reinigung zu einer Reduktion (einem Abtragen) der vorhandenen Keime, weshalb sie eine wesentliche Voraussetzung für den Erfolg der anschließenden Verfahren der Desinfektion und Sterilisation darstellt. Problematisch ist derzeit die Validierung von Reinigungsverfahren, da die Spezifikation für ein ,sauberes" Produkt nicht quantitativ definiert ist. Zwingend notwendig ist also eine ausreichende Klärung des tolerierbaren Grades der Restkontamination. Die Validierung des Gesamtprozesses der Aufbereitung gelingt nur, wenn für alle Teilprozesse quantitative Spezifikationen der zu erreichenden Ergebnisse vorliegen.

Eine weitere Problematik stellt in diesem Zusammenhang die Frage nach einer geeigneten Nachweismethode für den Reinigungserfolg dar. Die Einhaltung festgelegter quantitativer Spezifikationen muss sich auch im klinischen Alltag überprüfen lassen.

\section{ERGEBNISSE}

Der Gesetzgeber schreibt für die Aufbereitung von Medizinprodukten die Anwendung von validierten Verfahren vor.

Der Zweck der Validierung eines Verfahren besteht darin, eine Überprüfung der Eigenschaften auf eine Übereinstimmung mit der Spezifikation für jedes einzelne Produkt zu vermeiden. Ersatzweise geht man davon aus, daß die Einhaltung bestimmter festgelegter Verfahrensparameter in jedem Falle zu einem einwandfreien Ergebnis führt.

Ein solches Vorgehen wird bei Sterilisatoren seit Jahren angewendet. Wenn ein vorgegebener zeitlicher $\mathrm{Ab}$ lauf von physikalischen Größen (Druck, Temperatur, Dampfgehalt bzw. Gaskonzentration etc.) eingehalten wird, ist anzunehmen, daß die geforderte Reduktion der Keimzahl erreicht werden konnte.

Es wird also die nicht mögliche, da mit einem Verlust der Sterilität verbundene Prüfung jedes einzelnen Medizinproduktes durch die Überwachung von Verfahrensparametern ersetzt. Es erfolgt eine Parametrische Freigabe (parametric release) [5].

Die Notwendigkeit einer Bestimmung der Ausgangs- 
verschmutzung vor der Sterilisation (nach der Reinigung!) wird offensichtlich. Ist die Restbelastung durch Partikel (mögliche Fremdkörperreaktionen) oder Keime (Infektionen) nach der Reinigung zu hoch, so kann der Verfahrensschritt der Sterilisation auch bei Einhaltung der gewünschten Reduktion lebensfähiger Mikroorganismen trotz korrekter Bioindikator- und Dampfdurchdringungstests zu einem unsterilen Medizinprodukt am Ende der Aufbereitung führen.

Eine Normung, Vorgaben und Parameter für Reinigungsautomaten und Reinigungsverfahren befinden sich in der Entwurfsphase [6]. Folglich ist ein breiter Konsens bisher nicht gefunden worden.

Ein wesentliches Problem in diesem Zusammenhang ist der fehlende Konsens über ein geeignetes Nachweisverfahren für den Erfolg der Reinigung. Dabei geht es zum einen um eine Kombination aus einer Testverunreinigung und einer Nachweismethode die bei der Validierung der Reinigung für ein Produkt eingesetzt werden kann. Aber auch einfache Möglichkeiten der Routinekontrolle im klinischen Alltag sind umstritten.

\section{DISKUSSION}

Während die prinzipielle Notwendigkeit des Einsatzes von Automaten zur Reinigung im Sinne einer Validierbarkeit des Verfahrens allgemein anerkannt wird, gibt es keinen Konsens über die damit in Zusammenhang stehenden Detailfragen.

Bei der Validierung des Reinigungsverfahrens mußs eine definierte Testverunreinigung aufgebracht werden. Da es im Moment keinen Konsens über die Zweckmäßigkeit und Eignung in der Praxis eingesetzter Prüfverunreinigungen gibt, beschränkt sich der Normentwurf für Reinigungs- / Desinfektionsautomaten bisher auf eine leider unvollständige Aufzählung einiger, teilweise überlebter Varianten [6]. In Frage kommende Prüfanschmutzungen müssen insbesondere hinsichtlich ihrer Standardisierbarkeit und Aussagefähigkeit (Haftungsbedingungen auf dem Untergrund, Abtragbarkeit durch die Waschflotte usw. ), sowie Analysierbarkeit (Sensitivität, Spezifität, Reproduzierbarkeit und Robustheit der Methode hinsichtlich der zu prüfenden Stoffe) bewertet werden.

Dabei sind grundsätzlich zwei Herangehensweisen zu beobachten: Einerseits wird darauf abgezielt, möglichst nahe an den realen Verhältnissen zu bleiben, weshalb konsequenterweise die verschiedensten Blutvarianten eingesetzt werden. Dabei geht das Spektrum von frischem Humanblut bis zu Rindercitratblut oder Rinderserumalbumin mit/ohne Zusatz von Mucin oder weiteren möglichen Humananschmutzungen. Problematisch ist die fehlende Standardisierbarkeit von Blutanschmutzungen.

So werden andererseits definiert herstellbare Substanzen für die Prüfung favorisiert. Es finden mit dieser Argumentation Verunreinigungen wie Grießspudding, Hafermehl, Trockenkartoffelflocken, Senf etc. An- wendung. Eine interessante Lösung stellen in vitro hergestellte Prüfverunreinigungen dar, die Bestandteile des Humanblutes enthalten [3].

Für den Nachweis des Reinigungserfolges sind derzeit mehrere Protein-Nachweisverfahren in der Diskussion. An dieser Stelle nur zwei bedeutende Beispiele:

Die modifizierte OPA-Methode hat den Vorteil, bei in vivo eingesetzten Instrumenten angewendet werden zu können. Dabei werden die Instrumente mit einer SDS-Lösung (Natriumdodecylsulfat) gespült. Nach versetzen dieser Lösung mit Orthophthaldialdehyd ist ein photometrischer Nachweis des entstehenden Stoffes möglich. Das Ergebnis ist ein Extinktionswert.

Die Radionuklidmethode verfolgt einen grundsätzlich anderen Weg. Hierbei wird die Prüfanschmutzung mit einem radioaktiven Marker versehen. Nach erfolgter in-vitro-Anschmutzung und Aufbereitung kann eine eventuelle Restverschmutzung unter einer Gammakamera nachgewiesen werden. Dabei ist die mögliche Ortsauflösung des Ergebnisses ein Vorteil dieses Verfahrens. Das Ergebnis dieser Messungen ist die Angabe von counts.

Über die Korrelation der Ergebnisse beider Verfahren gibt es derzeit keine Erkenntnisse, jedoch laufen Untersuchungen, die hier eventuell einen Zusammenhang ergeben können.

Ziel des Einsatzes von Validierten Verfahren ist wie oben erwähnt die parametrische Freigabe der Aufbereiteten Produkte. Die wichtigsten zu diesem Zweck in einem Automaten zu überwachenden Parameter sind neben den Temperaturen die Spüldrücke, die Volumenströme des Reinigungsmediums durch die einzelnen Instrumente (eine Verstopfung in einem Instrument muß erkannt werden), die eingesetzte Menge von Reinigungsmitteln, der Leitwert des Mediums nach dem letzten Spülgang und die Applikation von Ultraschall (sofern vorhanden). Alle Parameter sind dabei in ihrem zeitlichen Verlauf zu dokumentieren.

\section{SCHLUSSFOLGERUNGEN}

Die Zielstellung kann nur in der konsequenten und vernünftigen Einführung validierter Reinigungsverfahren als entscheidender fehlender Baustein zu einer umfassenden Validierung des gesamten Aufbereitungsprozesses liegen.

In diesem Zusammenhang ist die gebräuchliche Einteilung in Einmal- und Mehrweginstrumente grundsätzlich in Frage zu stellen. Sinnvoller ist eine Unterscheidung in Instrumente mit und ohne produktspezifisch validiertem Aufbereitungsverfahren [4]. Ein derartiges Verfahren kann der Hersteller (im Falle der akzeptierten Wiederverwendbarkeit muß er es tun!), aber auch ein dritter Dienstleister entwickeln.

Voraussetzung für ein validiertes Reinigungsverfahren ist der Einsatz von Automaten. Speziell für die Aufbereitung von Instrumenten der Minimal Invasiven 
Chirurgie müssen Automaten entwickelt werden, an denen alle wesentlichen Parameter der Reinigung einstellund dokumentierbar seien müssen.

Es gibt bisher, wie oben erläutert kein allgemein anerkanntes Verfahren für die Routinekontrolle des Reinigungserfolges bei Instrumenten.

Selbst ein optimales Nachweisverfahren wird jedoch die Probleme nicht lösen. Es ist vielmehr damit zu rechnen, daß seine Anwendung viele Schwachstellen bei der Aufbereitung erst deutlich macht. Die sinnvolle und empfohlene [1][2] Entwicklung zu weitgehender Zerlegbarkeit der wiederverwendbaren Instrumente kann besonders bei komplizierten Geräten (z.B. Mehrfach-Clip-Applikatoren), aber auch bei einfachen Instrumenten wie Trokaren nicht die endgültige $\mathrm{Me}$ thode der Wahl sein. Eine Zerlegung ist sehr aufwendig und risikoreich. Es müßte im Sinne einer Rückverfolgung auch jedes Einzelteil eindeutig gekennzeichnet sein.

Ein hinreichend empfindliches und zuverlässiges Prüfverfahren vorausgesetzt, ist ein Instrument denkbar, dessen Lumina durch strömungstechnische Optimierung (keine Hinterschneidungen und unnötige Hohlräume, glatte Oberflächen, Spülanschlüsse, ...) ohne eine Zerlegung gereinigt werden kann. Viele heute auf dem Markt befindliche Instrumente sind weit von diesem Optimum entfernt! Hier liegt ein erhebliches Potential für eine Erhöhung der Qualität der Versorgung aber auch für eine Kostenreduzierung im Gesundheitswesen.

Insgesamt ist der Forschungs-, Entwicklungs- und Handlungsbedarf zur Verbesserung der Aufbereitung zu sterilisierender Chirurgieinstrumente, besonders mit Blick auf Minimal Invasive Techniken und die Endoskopie unübersehbar.

\section{Literatur}

[1] Fengler, Th. W.; Pahlke, H.; Kraas, E. : Instrumente für die endoskopische Chirurgie - neue Anforderungen an die Zentralsterilisation; Zentr. Steril. 2 1994: 9-20

[2] Fengler, Th. W.; Pahlke, H.; Kraas, E.: Eignungskriterien für das Laparoskopieinstrumentarium. Prospektive klinische Studie zu Funktion und Hygiene; chir. Praxis 55 1999: 5-16

[3] Pfeifer, M.: Standardisierte Testanschmutzung Blut - Zusammensetzung, Herstellung, Anwendung; Zentr. Steril., 61998

[4] Roth, K.;Heeg, P.;Reichl, R.;Cogdill, P.;Bond, W.: Qualitätssicherung bei der Aufbereitung von Zubehör für flexible Endoskope - Wie sauber sind gereinigte Instrumente wirklich?; Zentr. Steril. 2 1999; 84-96
[5] Schneider, P.M.: Requirements for Parametric Release of Steam Sterilized Products; Zentr. Steril. 1 2000; 20-23

[6] prDIN EN ISO $15883 \quad-\quad 1$ Reinigungs/Desinfektionsgeräte 